\title{
OBSERVATIONAL CONNECTIONS BETWEEN LBV'S AND OTHER STARS, WITH EMPHASIS ON WOLF-RAYET STARS
}

\author{
A. F. J. MOFFAT, L. DRISSEN and C. ROBERT \\ Département de Physique, Université de Montréal \\ C.P. 6128, Succ. "A" \\ Montréal, Québec H3C $3 J 7$ \\ Canada
}

\begin{abstract}
We suggest that the LBV mechanism is an essential step to "force" massive stars $\left(M(Z A M S) \geq 40 M_{\odot}\right)$ to finally enter the Wolf-Rayet (W-R) domain in the Hertzsprung-Russel diagram (HRD). Just as massive supergiants show increasing variability as they approach the Humphreys-Davidson (H-D)instability limit (horizontally in the HRD diagram), so the $W$ - $R$ stars show decreasing variability as they recede from the $H-D$ limit (at first horizontally into the WNL domain, then, with their high mass loss rates, plunging irreversably downwards as ever hotter, smaller and fainter, strong-line W-R stars). Among the W-R stars, the luminous WNL subtypes (especially WN8) are the most variable, probably as a consequence of blob ejection in the wind. The underlying mechanism which triggers this ejection is possibly related to wind instabilities and may thus be quite different from the source of variability in luminous supergiants or $L B V$ 's in quiescence, where photospheric effects dominate.
\end{abstract}

\section{Relatives of LBV's}

The most obvious immediate pre- and post-LBV objects must both be located below and to the left of the H-D limit, by default. Pre-LBV stars must be situated between the H-burning zero-age main-sequence (ZAMS) and the H-D limit; they obviously include the more luminous supergiants (SG) that have not yet shown any obvious LBV activity beyond quiescent microvariability (see below). They evolve essentially horizontally in the HRD, both before and after encountering the H-D limit (cf. Humphreys 1989).

The exact nature of post-LBV stars is more controversial. However, it does seem likely that after several major LBV eruptions, one should look among luminous stars with enhanced CNO-cycle abundances. The W-R stars are the most obvious candidates, in particular the most luminous ones, of type WNL (late-type W-R stars of subtypes WN6-9). After the WNL stage, W-R evolution progresses downwards in the HRD, as the stars lose more mass, shrink in size and head for the He-ZAMS (see below).

This brief review will be restricted to the blue relatives of LBV's, with emphasis on the W-R stars. Red SG's and the exotic OH/IR sources of high luminosity will not be considered here. The observational point of view is stressed, with minimal reference to theory. 


\section{Variability of Pre-LBV Supergiants}

A great deal of precise photometric data for SG's in the upper HRD has been accumulated and summarized by Maeder (1980) and later by Baade (1988). Figure 1 shows a résumé of Maeder's tabulated variability amplitudes, A, converted to standard deviation, $\sigma$, by assuming $A \simeq 3 \sigma$. Note that the level of variability increases towards higher luminosity for all spectral classes. (Note also that evolution of SG's occurs at $\sim$ constant bolometric luminosity, so that both the absolute visual magnitude $M_{v}$ and the spectral type change during this evolution.) The amplitudes for the most luminous SG's resemble the microvariations observed in LBV's during quiescence (cf. Lamers 1987). Some of these SG's may have already exhibited LBV-like eruptions.

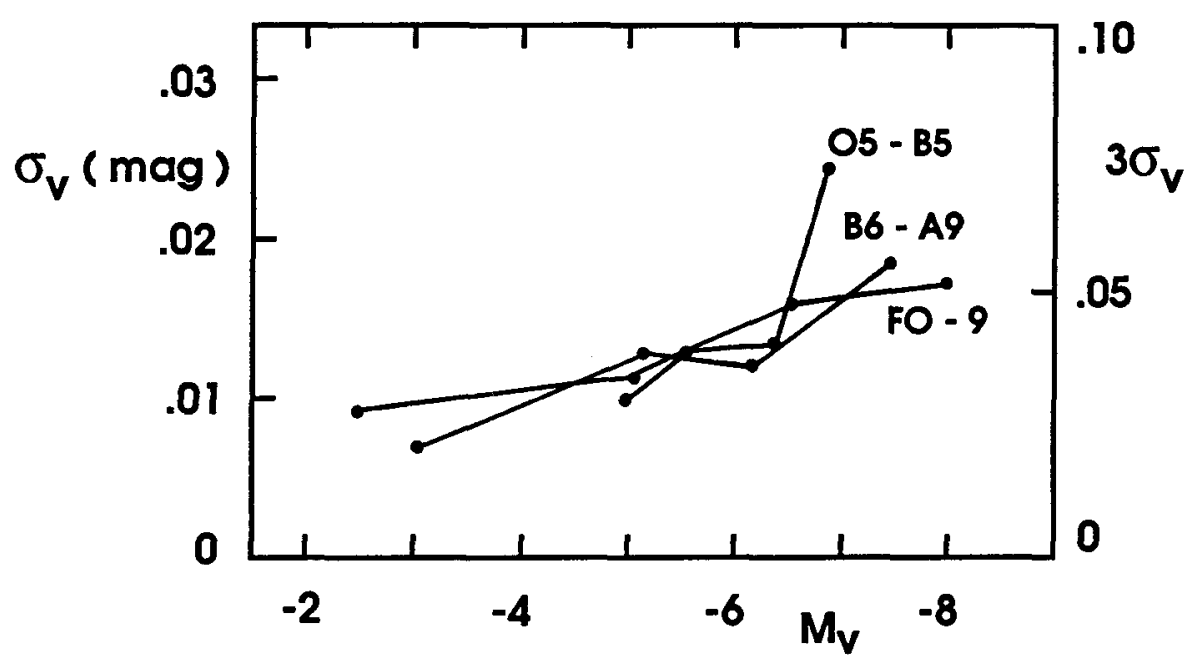

Figure 1. Variability of supergiants as a function of absolute magnitude (extracted from Maeder 1980)

\section{Variability of Post-LBV, Wolf-Rayet Stars}

W-R stars, with their intense emission lines and hot winds, occur in two main sequences: that of nitrogen and that of carbon (with an extension to oxygen for the latter). As for ordinary stars, they are binned into subclasses, in which higher values mean later (i.e. cooler) types. Calibration of W-R stars in Galactic open clusters and in the Large Magellanic Cloud, yields a very tight correlation between $M_{v}$ and subclass (Lundström and Stenholm 1984, updated by van der Hucht et al. 1988; Breysacher 1986). With mean bolometric correction $M_{b o l}-M_{v} \simeq-4.5 \pm 0.2$ for most W-R subclasses (Smith and Maeder 1988), this implies that the total luminosity also increases systematically from earlier to later WN or WC subtypes (e.g. $M_{b o l} \simeq-11$ for WN7 to -8 for WN4 or $M_{b o l} \simeq-9$ for WC8 to -8 for WC5).

$\mathrm{W}-\mathrm{R}$ stars also show remarkable similarity in variability behaviour compared to 
SG's. Figure 2 (adapted from Lamontagne and Moffat 1987) shows that both WN and WC stars exhibit greater variability for higher luminosity by an amount which is similar to the SG's at the same $M_{v}$. Variability of W-R stars in polarization shows similar behaviour (i.e. greater amplitude at higher luminosity) and is likely intimately related to the photometric behaviour (cf. Robert et al 1989a,b).

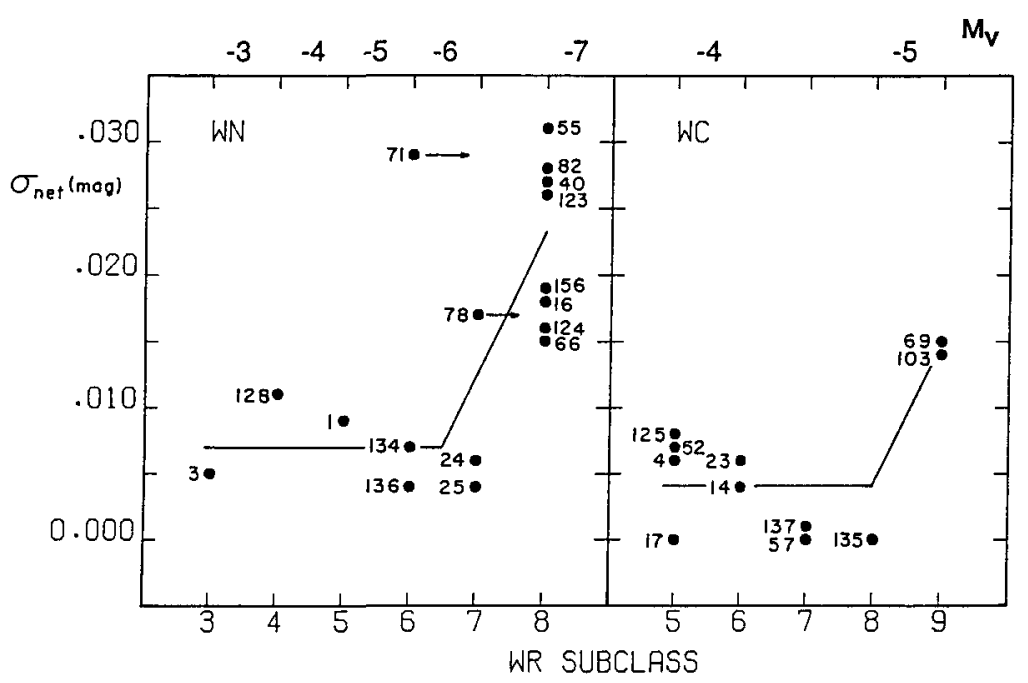

Figure 2. Variability of W-R stars as a function of subtype and absolute magnitude (from Lamontagne and Moffat 1987)

The mechanism that is most likely to cause the variability will be discussed in section 4. First, we look at the possibility of evolution among W-R stars of different subclass.

\section{The W-R Evolutionary Scenario}

Does each W-R star exist in its own right, independent of other W-R subtypes, or is there a pattern in the evolution, e.g. from one subtype to another? The answer to this question is still rather controversial but is fundamental and very relevant in establishing a connection or not with LBV's (and in the other direction, with supernovae!).

The approach we adopt here is mainly empirical, guided by a simple principle: we look for continuity of various parameters as a function of the most easily obtained quantity, the spectral subclass. We consider the following parameters:

(1) Mass: Although binary-estimated masses do scatter considerably even for a given subtype (the source for this may be intrinsic or spuriously related to the difficulty in eliminating the factor $\sin ^{3} \mathrm{i}$ ), there does exist a tight relation between mass ratio, $q=M(W R) / M(O)$, and subclass. Cooler W-R subtypes tend to have progressively higher ratios (Moffat 1981, 1982; Moffat et al. 1986; Moffat, 
Niemela and Marraco $1989=\mathrm{MNM}$ ). In particular, the WN6-7 stars have values of q close to unity, while all other observed subtypes (WC or WNE) generally have $\mathrm{q} \lesssim 0.5$, with $\mathrm{q} \sim 0.2$ for the hottest $\mathrm{W}-\mathrm{R}$ stars. Since binary mass transfer is probably not important in massive systems because of the fast winds (cf. MNM; Sybesma 1980), the mass ratio in W-R + O systems essentially gives a normalized measure of the amount of mass shed by the W-R star, regardless of the original mass. Note that the original mass ratio must have been $\gtrsim 1$ (values differing greatly from unity are excluded by observations of $\mathrm{O}+\mathrm{O}$ binaries: Garmany, Conti and Massey 1980). The fact that the spectral types of the $\mathrm{O}$-companions in W-R binaries show no correlation with W-R spectral subclass implies that all W-R stars can evolve from any massive star (normally $\gtrsim 40 M_{\odot}$; cf. Humphreys, Nichols and Massey 1985).

(2) Luminosity: We already saw in section 2 that the later the W-R subtype, the brighter the star. Especially the WNL stars are the brightest.

(3) Spatial distribution: While WNL stars generally occur in any part of the Galactic disk where active star formation has taken place, other W-R subtypes show a fairly clear frequency dependence on galactocentric distance (van der Hucht et al. 1988).

(4) Line profiles: While WC stars show a clear trend of very broad emission lines in the hottest subtypes to narrow lines in the coolest, the correlation exists too but is noisier for the WN stars. One should probably distinguish broad- from narrow-line WN stars of the same subtype (cf. Hiltner and Schild 1966; Walborn 1974).

(5) Ages: On the basis of the morphology, sizes and kinematics of the ring nebulae surrounding a number of W-R stars, Chu, Treffers and Kwitter (1983) demonstrated that WC stars are generally older than WN stars and WNE are older than WNL.

(6) Abundances: Among the WN stars, it has been known for some time that the WNL stars often contain hydrogen in their winds, while WNE stars are $\mathrm{H}$ poor (e.g. Smith 1973). Among the WC stars, recent evidence also yields a progression of higher C/He from cool to hot WC (Smith and Hummer 1988; but cf. Torres 1988 and de Freitas Pacheco and Machado 1988).

(7) Variability: As noted in section 2, there is a clear trend in continuum light and polarization variability such that cooler (especially WN8) stars reveal larger amplitudes.

(8) Core radii: Although not directly visible in most W-R stars, the stellar radii (where the radial wind velocity is near zero) have been extracted from eclipsing binaries in a few cases. The trend is that cooler subtypes tend to be larger (e.g. $11 R_{\odot}$ for the WN7 star CQ Cep (Leung, Moffat and Seggewiss 1983) and $\sim 3 R_{\odot}$ for the WN5 star in V444 Cyg (Cherepashchuk, Eaton and Khaliullin 1984)). It is assumed that the remaining subtypes follow a continuous trend in radius, as does the luminosity or mass ratio.

All of the above trends, together with the high mass loss rates, favour subtype evolution from cooler to hotter subtypes within each sequence. Our interpretation is as follows: all W-R stars start as WNL, the most massive, luminous and least evolved of all subtypes. As the strong wind peels off the outer layers, the surface abundance gets more exotic with time and the core radius shrinks. Transfer from 
WN to WC, basically a surface phenomenon, will occur at a given WN-subtype which depends on the initial metallicity $Z$ (Moffat 1981, 1982). In short, extreme mass loss forces W-R stars to evolve downwards (fainter) and to the left (hotter) in the HRD, more or less toward the He-ZAMS. This is no surprise, since W-R stars are Heburning, with H-rich surface impurities that diminish with time. With typical mass loss rates $\dot{M} \simeq 4 \times 10^{-5} M_{\odot} y r^{-1}$ (e.g. Schmutz and Hamann 1986), the total mass lost in the W-R phase (mean lifetime $\mathrm{t} \sim 5 \times 10^{5}$ yr: Maeder and Meynet 1987) is $\Delta M=\dot{M} t \sim 20 M_{\odot}$ (variations of \pm a factor 2 are possible), which is entirely compatible with the above interpretation. For example, a W-R star in a binary, going from $\mathrm{q}=1.0$ to 0.2 , will lose $\Delta \mathrm{q} \times M_{\circ} \approx 24 M_{\odot}$ for an average $M_{\odot}=30 M_{\odot}$ O-type companion.

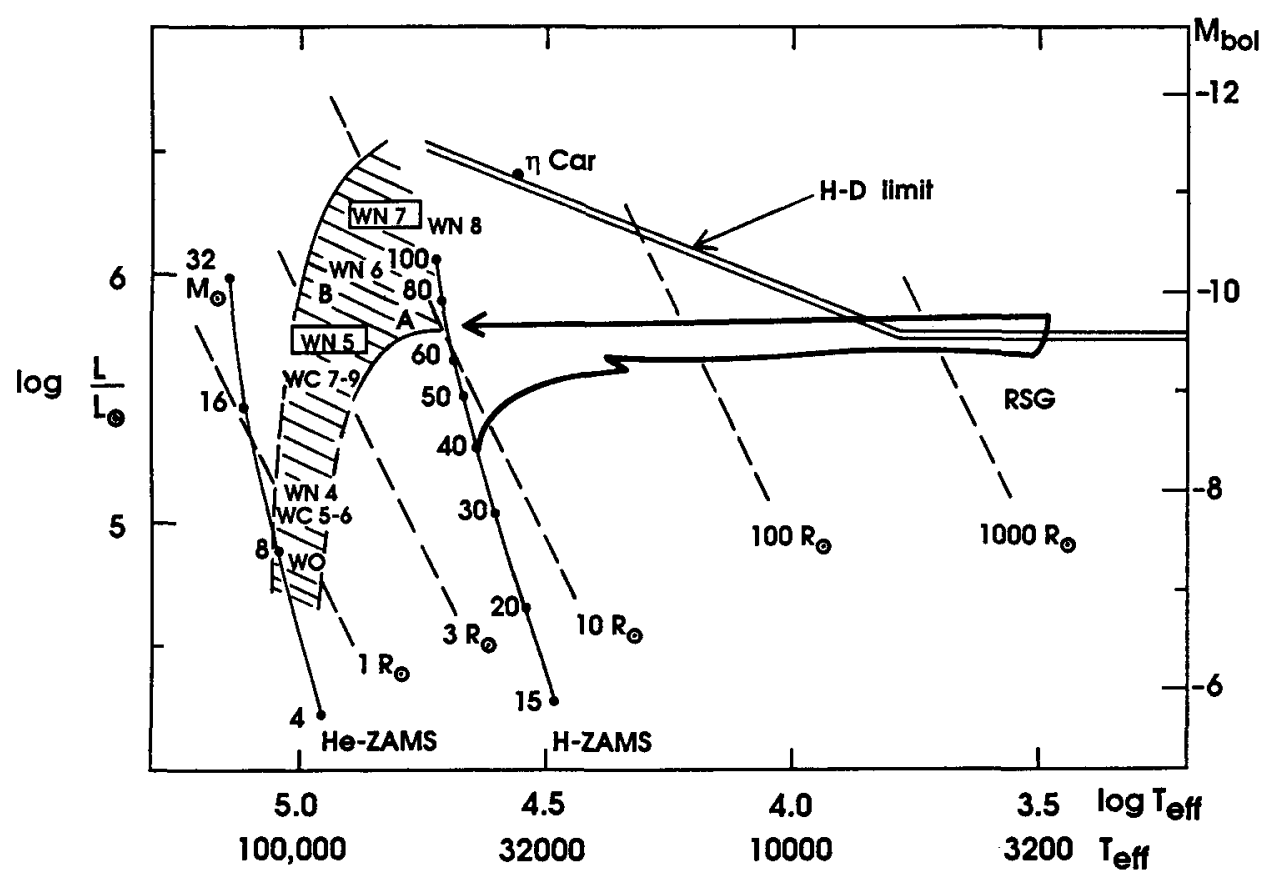

Figure 3. Average positions of W-R stars of different subclass in the HR diagram. The positions of the framed subtypes are constrained by direct eclipse observations of stars of the corresponding type. The hatched, funnel-shaped region allows for the observed spread in $M_{b o l}$. The evolutionary track of a $40 M_{\odot} Z A M S$ star from Maeder and Meynet (1987) is shown for comparison.

Based on the above arguments (especially that hotter W-R stars tend to be smaller in core radii and less luminous), Figure 3 shows our best empirical interpretation of 
$\mathrm{W}-\mathrm{R}$ evolution in the HRD. Note the rough distinction between narrow-line (A) and broad-line(B) WN stars. Both are supposed to have similar L (thus also $M$ ), but the WN-B stars have higher $v_{\infty}$. This makes their radii smaller, assuming the correlation between $v_{\infty}$ and escape velocity of Abbott (1982) for hot winds: $v_{\text {esc }} \simeq$ $v_{\infty} / 3=\sqrt{2 G M(1-\Gamma) / R}$, also applies to W-R stars ( $\Gamma$ is the ratio of radiation to gravitation force). With smaller radii but equal $\mathrm{L}$ (and $\Gamma$ ?), the WN-B stars will thus be hotter than WN-A stars. Judging from their approximate, deduced position in Figure 3, WN-B stars probably evolved from more massive progenitors than did WN-A stars.

Figure 3 shows that all W-R stars are extracted, initially as WNL, out of the region of the HRD where most LBV's are found (M(ZAMS) $\gtrsim 40 M_{\odot}$; cf. Humphreys 1989). Independant information reveals that the progenitor masses of W-R stars must also have been $\gtrsim 40 M_{\odot}$ (cf. Humphreys, Nichols and Massey 1985). After WNL, they evolve down a progressively narrower, funnel-shaped path toward the hotter HeZAMS. Of course, this scenario is rough and needs some fine-tuning, especially at the faint end. Note that the positions of the W-R subtypes in Figure 3 refer to the cores and not necessarily to $\tau \sim 1$, which often occurs in the winds.

Figure 3 also implies that, in order for a massive star to reach the W-R domain, it first has to encounter the H-D instability barrier (as an LBV), causing it effectively to "bounce" back eventually to the left of the HRD. Once the W-R domain is reached, the star will not return to the H-D limit. Stars well below $\sim 40 M_{\odot}$ do not generally encounter this limit and probably do not become W-R stars either, in general. Stars just below $40 M_{\odot}$ may become "red LBV's" (e.g. OH/IR variable red SG's) and then W-R. Note that there exist several apparently genuine LBV's of relatively low luminosity (e.g. R66, AE And, M31/var 15: Lamers 1987) with deduced masses in the range $\sim 20-30 M_{\odot}$; if the data for these stars are reliable, then their evolutionary future is not clear.

\section{Mechanism of W-R Star Variability: Relevance to LBV's}

Among W-R stars, those of subtype WN8 (and WN9?) appear to be the closest to LBV's with respect to their high degree of variability, coupled with their relatively narrow emission lines and their high luminosity (although not higher than WN7 stars, which, curiously, are more stable). WN8 stars are also closer to LBV's in the HRD (cf. Figure 3). However, WN8-9 stars show some unusual characteristics. For example, none of 9 Galactic and LMC WN8-9 stars monitored for RV variations show any sign of being a W-R + O binary (Moffat 1989). This contrasts starkly with the 26 monitored WN6-7 stars which are 58\% W-R + O. Also, WN8-9 stars tend to avoid clusters, unlike WN6-7 stars, and some appear to be runaways. Possibly WN8-9 stars are slightly lower mass WNL stars associated with lower mass LBV's (cf. Lortet and Testor 1988) that occur more towards the red part of the HRD. On the other hand, it is probably no coincidence that the massive LBV $\eta$ Car occurs in a young cluster together with (more massive) WN7 stars.

In order to probe the nature of the variations in W-R stars more deeply, it is essential to carry out high resolution, high $\mathrm{S} / \mathrm{N}$, time-resolved $(\leq 30 \mathrm{~min})$ spectroscopy. This allows the additional advantage over continuum studies of being able to explore the velocity domain (hence also the spatial domain if both domains are correlated, say, by a global wind velocity law). Intense monitoring of a few bright W-R stars has 
been carried out (Moffat et al 1988; Robert, Drissen and Moffat 1989a; McCandliss and Bohannan 1989; Koenigsberger and Massey 1989), but only for the subtypes WN5-6, WC7-8 and for a limited selection of spectral lines. In particular, the first two references reveal strong evidence for random, radial acceleration of blobs which are carried along with the general wind. The study of a symmetric, unblended emission line in this case allows one to see optically thin, emitting blobs being ejected in all directions.

What triggers the blobs? There are two main contenders: (1) non-radial pulsations (Vreux 1985) or (2) stochastic radiative wind instabilities (Owocki, Castor and Rybicki 1989). The former mechanism has been shown to be unlikely to occur in W-R stars (Matthews and Beech 1987; Cox and Cahn 1988; however, cf. Noels and Scuflaire 1987). Direct observational confirmation will be difficult, however, due to the filtering action of the wind. The latter mechanism seems very attractive but has one problem: the theory predicts that fast winds should be less stable, while the observations of W-R stars seem to indicate the opposite, namely that inhomogeneities appear to form more readily and/or have more time to grow in cooler, slower winds.

Beyond the microvariation amplitudes of WN8 stars and LBV's in quiescence, the similarity between W-R stars and LBV's ends. First, the timescales for microvariations are quite different, being $\sim 1 \mathrm{~d}$ in WN8 stars and $\geq 10 \mathrm{~d}$ in LBV's. Also, WN8 stars are not known to show outbursts. Their variability is a wind phenomenon, whose relation to the underlying star, if any, is unknown. In LBV's, the variability is mainly a photospheric effect at the stellar surface. The same difference applies between W-R stars and the SG progenitors of LBV's.

\section{Conclusions}

In a sense, LBV's represent the peak of a mountain. Stars climbing up to the top as SG's become more and more variable. Stars descending from the peak into the $\mathrm{W}-\mathrm{R}$ domain become less and less variable. Once the star enters the W-R zone, it has reached the point of no return.

The overall scenario for stars of initial mass above $\sim 40 M_{\odot}$ may be as follows:

$\rightarrow$ Supergiant (looping horizontally in the HRD)

$\rightarrow$ LBV (several times, depending on the mass loss and not just in the blue $\rightarrow$ WNL part of the HRD-cf. Humphreys 1989)

(Z low) WNE - - $\rightarrow$

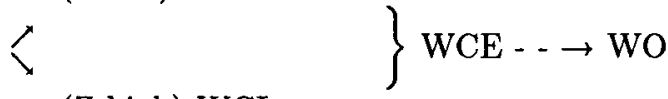

(Z high) WCL $\longrightarrow$

$\rightarrow$ Supernova of type Ib, probably as WNE, WCE or WO subtypes (cf. Ensmann and Woosley 1988, whose models of SN Ib light curves show a preference for $\mathrm{W}-\mathrm{R}$ progenitors of mass $\sim 4-7 M_{\odot}$, corresponding to the hotter W-R subclasses).

Some stars in the range $30-40 M_{\odot}$ might become LBV-like red SG's and later proceed to the W-R domain. An analogy of the above scenario $\left(M_{i} \gtrsim 40 M_{\odot}\right)$ can be drawn with low mass stars $\left(M_{i} \lesssim 8 M_{\odot}\right)$, which rise far up the asymptotic branch near to the position of the highly variable OH/IR stars, eject planetary nebulae, pass 
quickly to the left in the HRD and descend down the Harman-Seaton cooling sequence to the white dwarf domain.

\section{References}

Abbott, D. C. 1982, Ap. J., 259, 282.

Baade, D. 1988, in "O stars and Wolf-Rayet stars", eds. P. S. Conti and A. B. Underhill, NASA SP-497, p. 137.

Breysacher, J. 1986, Astron. Ap., 160, 185.

Cherepashchuk, A. M., Eaton, J. A. and Khaliullin, Kh. F. 1984, Ap. J., 281, 774.

Chu, Y. H., Treffers, R. R. and Kwitter, K. B. 1983, Ap. J. Suppl., 53, 937.

Cox, A. N. and Cahn, J. H. 1988, Ap. J., 326, 804.

Ensman, L. M. and Woosley, S. E. 1988, Ap. J., 333, 754.

de Freitas Pacheco, J. A. and Machado, M. A. 1988, A. J., 96, 365.

Garmany, C. D., Conti, P. S. and Massey, P. 1980, Ap. J., 242, 1063.

Hiltner, W. A. and Schild, R. E. 1966, Ap. J., 143, 770.

van der Hucht, K. A., Hidayat, B., Admiranto, A. G., Supelli, K. R. and Doom, C. 1988, Astron. Ap., 199, 217.

Humphreys, R. M. 1989 , these proceedings.

Humphreys, R. M., Nichols, M. and Massey, P. 1985, A. J., 90, 101.

Koenigsberger, G. and Massey, P. 1989, these proceedings.

Lamers, H. J. G. L. M. 1987, in "Instabilities in Luminous Early Type Stars", eds. H. J. G. L. M. Lamers and C. W. H. de Loore, (Reidel:Dordrecht), p. 99.

Lamontagne, R. and Moffat, A. F. J. 1987, A. J., 94, 1008.

Leung, K. C., Moffat, A. F. J. and Seggewiss, W. 1983, Ap. J., 265, 961.

Lortet, M.-C. and Testor, G. 1988, Astron. Ap., 194, 11.

Lundström, I. and Stenholm, B. 1984, Astron. Ap. Suppl. Ser., 58, 163.

Maeder, A. 1980, Astron. Ap., 90, 311.

Maeder, A. and Meynet, G. 1987, Astron. Ap., 182, 243.

Matthews, J. M. and Beech, M. 1987, Ap. J., 313, L25.

Mc Candliss, S. R. and Bohannan, B. 1989, these proceedings.

Moffat, A. F. J. 1981, in "Effects of Mass Loss in Stellar Evolution", IAU Coll. No. 59, eds. C. Chiosi and R. Stalio, (Reidel:Dordrecht), p. 301.

Moffat, A. F. J. 1982, in "Wolf-Rayet Stars: Observations, Physics, Evolution", IAU Symp. No. 99, eds. C. W. H. de Loore and A. J. Willis (Reidel:Dordrecht), p. 515.

Moffat, A. F. J. 1989, in preparation.

Moffat, A. F. J. , Vogt, N., Paquin, G., Lamontagne, R. and Barrera, L. H. 1986, A. J., $91,1386$.

Moffat, A. F. J. , Drissen, L., Lamontagne, R. and Robert, C. 1988, Ap. J., 334, 1038.

Moffat, A. F. J. , Niemela, V. S. and Marraco, H. 1989, Ap. J., submitted.

Noels, A. and Scuflaire, R. 1987, in "Instabilities in Luminous Early Type Stars", eds. H. J. G. L. M Lamers and C. W. H. de Loore, (Reidel:Dordrecht), p. 213.

Owocki, S. P., Castor, J. I. and Rybicki, G. B. 1989, these proceedings.

Robert, C., Drissen, L. and Moffat, A. F. J. 1989a, these proceedings.

Robert, C., Moffat, A. F. J. , Bastien, P., Drissen, L. and St.-Louis, N. 1989b, Ap. $J .$, submitted.

Schmutz, W. and Hamann, W.-R. 1986, Astron. Ap., 166, L11.

Smith, L. F. 1973, in "Wolf-Rayet and High Temperature Stars", IAU Symp. No. 
49, eds. M. K. V. Bappu and J. Sahade, (Reidel:Dordrecht), p. 15.

Smith, L. F. and Hummer, D. G. 1988, M. N. R. A. S., 230, 511.

Smith, L. F. and Maeder, A. 1988, preprint.

Sybesma, C. H. B. 1986, Astron. Ap., 168, 147.

Torres, A. V. 1988, Ap. J., 325, 759.

Vreux, J.-M. 1985, P. A. S. P., 97, 274.

Walborn, N. 1974, Ap. J., 189, 269.

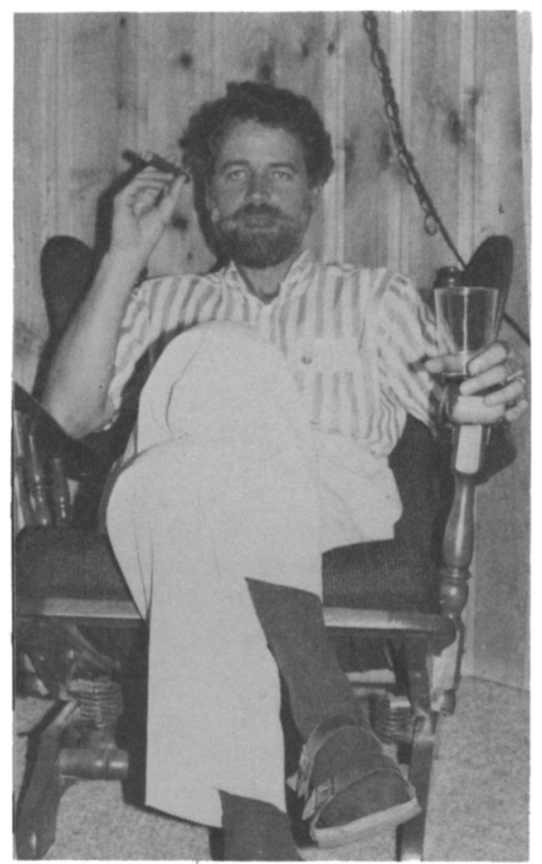

Tony Moffat 


\section{DISCUSSION}

Koenigsberger: The winds of WNL stars are said to be less optically thick than those of WNE and WC stars. Is it possible that WNE and WC stars are intrinsically just as variable as WNL's and we just don't see it?

Moffat: Yes, this might be so. However, in the context of radiative instabilities à la Rybicki/Owocki, one would expect the instabilities (= blobs?) to grow as they propagate outwards. Since we are seeing the winds farther out for WC/WNE stars than for WNL stars (i.e., the $\tau_{e s} \sim 1$ levels), one might expect the amplitude to be even larger in WC/WNE stars, contrary to the observations.

Sreenivasan: I suspect that one cannot make a direct connection between the blobs that you observe and the presence/absence of radial/non-radial pulsations, because the regions that you see are so far out in the wind. Radial pulsations in massive stars may be indicated in the work of Maeder and of Cox and Cahn. The latter authors did not find non-radial pulsations in WR stars, but Kirbiyak and others claimed they did. None of these workers studied the effects of rotation. Non-radial pulsations could be present in WR stars because of differential rotation. Lee and Saoi found non-radial pulsations in $10 M_{\odot}$ models. The blobs are presumably due to effects such as those considered by Owocki et al. in the wind. Non-radial pulsations could affect mass-loss rates but cannot by themselves cause the formation of blobs.

Moffat: Indeed, the connection has yet to be made. However, if rotation or pulsation do play a role in WR stars, this is certainly not obvious judging from the random nature of the observed wind variations.

Fried jung: How close are Wolf-Rayet stars now believed to be to the Eddington limit? Can you have an optically thick wind when such a star is not very close to it?

Moffat: I think that most WR researchers agree that WR stars must be close to their their Eddington limits. The question is what opacity $\kappa$ to take for the base of the wind to yield $L_{\mathrm{Edd}}=4 \pi G c M / \kappa<L$ ? Certainly electron scattering opacity alone is not sufficient, since the gas is not fully ionized (cf. arguments for LBV's given by Lamers \& Fitzpatrick 1988, Astrophys.J. 324, 279).

Conti: The "funnel" analogy for the end phases of massive star evolution appears to be a good one, although I am not convinced that a unique WR subtype can be identified at every point "downstream." I would like to take issue with your inferred relationship between variability and absolute magnitude $M_{\mathrm{V}}$. Clearly there is a correlation with spectral subtype in both WN and WC stars, as you and your coworkers have so ably pointed out. But most of the purported relationship to $M_{\mathrm{V}}$ depends on the relatively bright $M_{\mathrm{V}}$ assigned to WN 8 stars. The absence of WN 8 stars in H II regions implies that they do not originate from the most massive progenitors, nor from the most luminous stars. These WN 8 objects might well come from certain LBV's, whose spatial distributions are similar. To demonstrate a dependence of variability on $M_{\mathrm{V}}$ you need to study stars of similar type but with different $M_{\mathrm{V}}$ 's to see if the variability depends on brightness, independent of radius.

Schmutz: The values you used for the basic stellar parameters of the WR stars are more or less guesses. However, reliable parameters are now determined for $30 \mathrm{WR}$ stars (Schmutz et al., Astron. Astrophys. in press). The important point of these new results is that for about half of the objects there is no difficulty in determining the temperatures of the stars themselves -- their continuum formation layers are not extended. For another $1 / 4$ of the WR stars the correction between the $\tau=2 / 3$ level 
and the stellar surface is not large. For the remaining $1 / 4$ of the stars there is a large uncertainty; but for the majority of the WR stars there is no longer a large uncertainty in their basic stellar parameters.

Moffat: The stellar parameters that we used are based on observations, e.g., $L$ from WR stars in clusters, masses from binaries, and radii from the few known eclipsing binaries (then $L=4 \pi R^{2} T_{\text {eff }}$ gives $T_{\text {eff }}$ ). Where there are gaps $I$ have drawn from arguments of continuity with spectral subclass and Occam's razor. In any case, your theoretical results agree fairly well with the observed values.

Van der Hucht: One of your favorite WR binaries, HD 193793, has an O4 V secondary. Maeder's evolutionary models cannot make a WC7 with the same age as an O4 V star. It is premature to rule out mass transfer: binary component evolution may be different from single-star evolution. This jeopardizes your scenario for subtype evolution.

Moffat: HD 193793 has the longest known period of any known WR + $O$ binary (period 7.9 years!). Even with an eccentricity $\sim 0.7$, it is difficult to see how even case $B$ mass transfer could have taken place. Even if it did, it should have been highly nonconservative as a result of the extremely hot, dense, fast stellar winds (either $\mathrm{O}+\mathrm{O}$, $\mathrm{BSG}+\mathrm{O}, \mathrm{RSG}+\mathrm{O}$ or $\mathrm{WR}+\mathrm{O}$ ). Perhaps our understanding of stellar evolution related to spectral types is deficient! More generally, if WR stars in binaries do behave differently from single WR stars, this is not empirically obvious on the basis of spatial and spectral subtype distributions.

Vanbeveren: If observations of WR binaries reveal secondaries with spectral classes as early as $07,06,05 \ldots$, then one needs mass transfer in order to explain this (both components must have the same age).

Moffat: For O-supergiants I would say there is less of a problem. For main-sequence $\mathrm{O}$-stars, I agree that there appears to be a problem with the ages. However, if mass transfer does take place, then it becomes difficult to explain eccentric $W R+O$ binaries. Also, I find it difficult to believe that significant mass transfer took place in the 7.9-year WC7 + O4V binary HD 193793, with its large separation and high terminal wind speeds. It would be nice to see some hydrodynamical calculations. Finally, I wonder how good our knowledge is of the spectral-type evolution of the most massive stars.

Gallagher: In considering the role of binary evolution, you also have to include the possible effects of embedded binary cores during the pre-WR evolutionary phases. For example, wouldn't a system like CQ Cep almost certainly have had a common envelope during its supergiant and LBV evolutionary phases? If so, this would have influenced its observable properties, even within your assumption of minimal mass tranfer within the binary.

Moffat: CQ Cep is a poor example (SB1) but nevertheless the only known eclipsing system involving a WN7 star. I assume that how it became WN7 is less important in this context than that it yields an estimate of the radius of a WN7 star $\left(11 R_{\odot}\right.$ according to Levy et al. 1983). The WN7 phase is only the beginning, when mass loss is relatively minor compared to subsequent WR phases (WNE and/or WC), in which a common envelope in the sense that you mentioned is not very relevant.

Willis: Returning to the variability aspects: Have you any estimate of the mass of an individual blob, and the total mass ejection rate involved in the variability?

Moffat: On the basis of the power contained in the standard deviation profile for pure emission lines, we can say that typically a few percent of the wind is in the form of visible propagating blobs. This fraction appears to vary with subclass (it's 
higher for cooler stars) and ionization potential (lower-ionization lines are generally more variable). However, it may be that the whole wind consists of discrete blobs, whose size-spectrum we are only sampling at the massive end. At any given time there are only a few of the largest blobs; thus, to find the mass in the blobs, you can take your favorite wind model and convert the observed fraction into mass.

Van der Hucht: Do you think it is realistic to associate blob ejection which occurs at a few $R *$ with the clumpy structure of RCW 58, whose radius is a few parsecs?

Moffat: Since the central star HD 96548 (WN8) ejected the material that we see in RCW 58 (cf. Chu et al. 1983, Astrophys.J.Suppl. 53, 937), it is tempting to make some kind of association between the clumpy structure of RCW 58 and one of the intrinsically most variable WR stars in the sky. Possibly the present clumps are related to very large blobs ejected in the past. Note that older WR ring nebulae are generally much smoother after interaction with the interstellar medium.

Langer: I have a question concerning the linear decrease in luminosity for WN stars from late to early spectral types that you reported. Do you think one could conclude that the subluminous LBV's (e.g. R 71) must evolve directly into early-WN stars, skipping the WNL phase?

Moffat: As noted by Humphreys (1987, Lunteren meeting, p. 10), most LBV's are brighter than $M_{\text {bol }} \sim-10$. The few fainter examples presumably originated as stars with lower initial masses $\left(40-50 M_{\odot}\right)$. Such stars can still become WR stars first as WNL's, at the lower edge of my funnel entrance. An observational bridge of continuity is lacking for such LBV's to go directly to the very broad-line WNE stars. [Note also that there are discrepancies in the literature and problems with the luminosities: e.g., Lamers in the Lunteren meeting, p. 101, gave $M_{\text {bol }}=-10.6$ for R 71 . Leitherer (these proceedings) gives a value of -8.8.]

Hillier: Firstly, I believe that many of the conclusions drawn by Tony about WR stars were highly optimistic. Secondly, a lot of high-quality observations concerning variability have appeared at this colloquium. I would, however, like to see more work attempting to correlate the effects of "ejected blobs" on different spectral lines. In particular, the work of Hamman \& Schutz, and by myself, indicates that the He II lines should be affected before the HeI lines.

Moffat: Sometimes one has to smooth rough corners in order to assess the global scene (forest $v s$. trees). Indeed we (and others) are very aware of the stratification in WR winds, such that higher ionization lines formed generally at smaller radii in the wind should be affected first by blob ejection. But they should also show less variability as they occur earlier in the growth phase, if the Rybicki/Owocki scenario is correct. If telescope allocation committees had been more generous, we might have been able to answer this question observationally by now! 